\title{
Fluid flow simulation in a double L-bend pipe with small nozzle outlets
}

\author{
A. Rigit ${ }^{1}$, J. Labadin ${ }^{2}$, A. Chai ${ }^{1}$ \& J. Ho ${ }^{1}$ \\ ${ }^{I}$ Faculty of Engineering, University Malaysia Sarawak, Sarawak, \\ Malaysia \\ ${ }^{2}$ Faculty of Computational Science and Information Technology, \\ University Malaysia Sarawak, Sarawak, Malaysia
}

\begin{abstract}
The results of fluid flow simulation in a double L-bend pipe with small nozzle outlets are presented in this paper. The pipe geometry represents a sparger for a mixing process in a tank. The flow simulation was performed with a commercially available computational fluid dynamics package, Star-CD. The effects of the L-bend and small nozzle outlets on the velocity and pressure distributions in the pipe are discussed. The discussion will lead to an improved design of the sparger with the objective of obtaining a uniform fluid discharge from the nozzle outlets.

Keywords: flow simulation, computational fluid dynamics, velocity and pressure distributions.
\end{abstract}

\section{Introduction}

The primary process in manufacturing computer hard discs is the process of coating nickel sulphamate solution onto the discs. The nickel solution is normally fed into a mixing tank via a double L-bend pipe, which represents a sparger with small nozzle outlets. However, the coating process may be affected by non-uniform distribution of the nickel solution inside the mixing tank, resulting in an uneven nickel coating thickness at the discs surface. It is therefore desirable to have a uniform fluid discharge from the pipe nozzle outlets in order to obtain a uniform distribution of the nickel solution inside the mixing tank. The variables that may be changed in order to get the optimum results would be the pipe geometry, design or even the control of pressure of the fluid flow as 
previously discussed by Morrison [1] and Aroussi et al. [2, 3] on CFD and experimental studies for a gas phase flow in a double elbows pipe problem.

The objectives of this paper are to present the fluid flow visualization in the pipe and to decide which variables that should be changed in order to obtain a uniform fluid discharge from the nozzle outlets.

\section{Computational modelling}

\subsection{The configuration examined}

The computational fluid dynamics (CFD) explores the effects of a double $90^{\circ} \mathrm{L}$ bend and a series of equally spaced small nozzles along one side of the pipe horizontal arm on the fluid flow. The configuration used in the study is shown in Fig. 1. The pipe geometry is a double L-bend configuration with both elbows perpendicular to each other. The length of the smooth pipe is modelled to be equivalent to 20 times the diameter of the pipe. This double L-bend configuration also replicates many pipe structures present in a range of pipe networks.

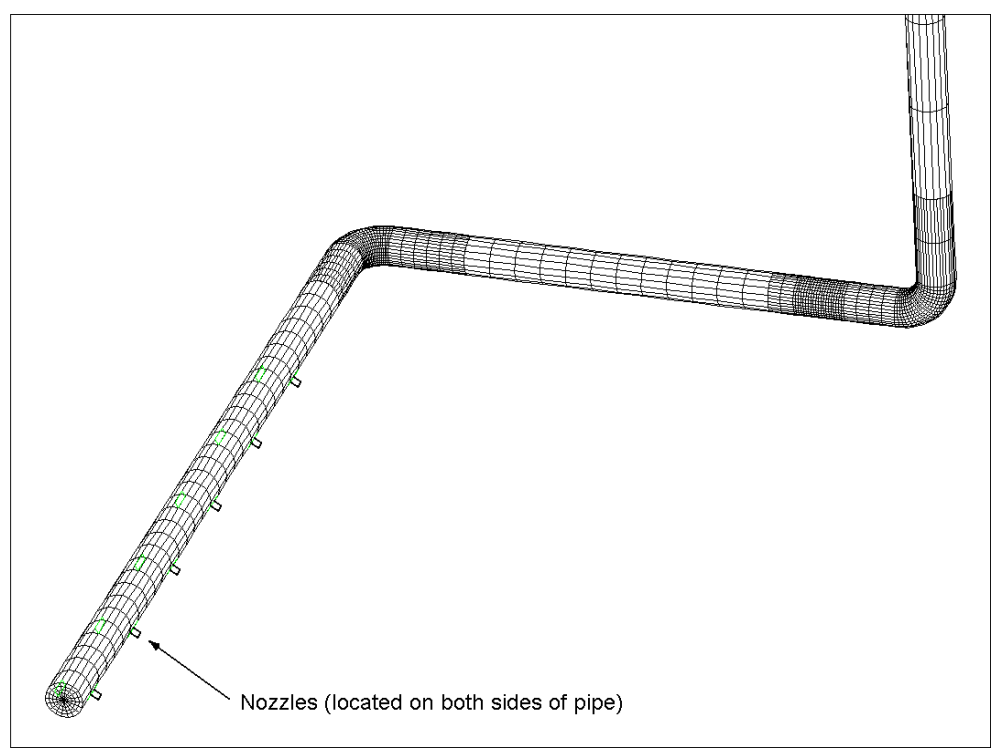

Figure 1: The configuration of the double L-bend pipe with series of nozzles located after the $2^{\text {nd }}$ bend.

\subsection{The CFD model}

The flow predictions were carried out using a commercially available CFD package, Star-CD 3.15. The solid geometries were modelled using SolidWorks, 
which are then imported into ProStar of Star-CD. The grid generation and meshing were created using manual-generation of vertices and splines.

Star-CD provides various differencing scheme in the package in order to produce fluid simulation that best describes the problem nature. The centraldifferencing (CD) scheme was chosen over the other schemes such as upwinddifferencing (UD) and self-filtered central differencing (SFCD) since the CD scheme:

a) is of higher order scheme (second-order accuracy),

b) interpolates linearly on the nearest neighbouring values, and

c) produces less numerical diffusion;

SFCD blends UD and CD together and thus making it a second-order accurate. However, this scheme was not chosen due to the fact that it has the possibility of generating additional non-linearity. An example of the CD scheme being applied to flow simulation is described by Mendonça et al. [6].

The mass and momentum conservation equations solved by Star-CD for general incompressible and compressible fluid flows and a moving coordinate frame (essentially, the Navier-Stokes equations) are, in Cartesian tensor notation [7]:

$$
\begin{aligned}
& \frac{1}{\sqrt{g}} \frac{\partial}{\partial t}(\sqrt{g} \rho)+\frac{\partial}{\partial x_{j}}\left(\rho \tilde{u}_{j}\right)=s_{m} \\
& \frac{1}{\sqrt{g}} \frac{\partial}{\partial t}\left(\sqrt{g} \rho u_{i}\right)+\frac{\partial}{\partial x_{j}}\left(\rho \tilde{u}_{j} u_{i}-\tau_{i j}\right)=-\frac{\partial p}{\partial x_{i}}+s_{i}
\end{aligned}
$$

where

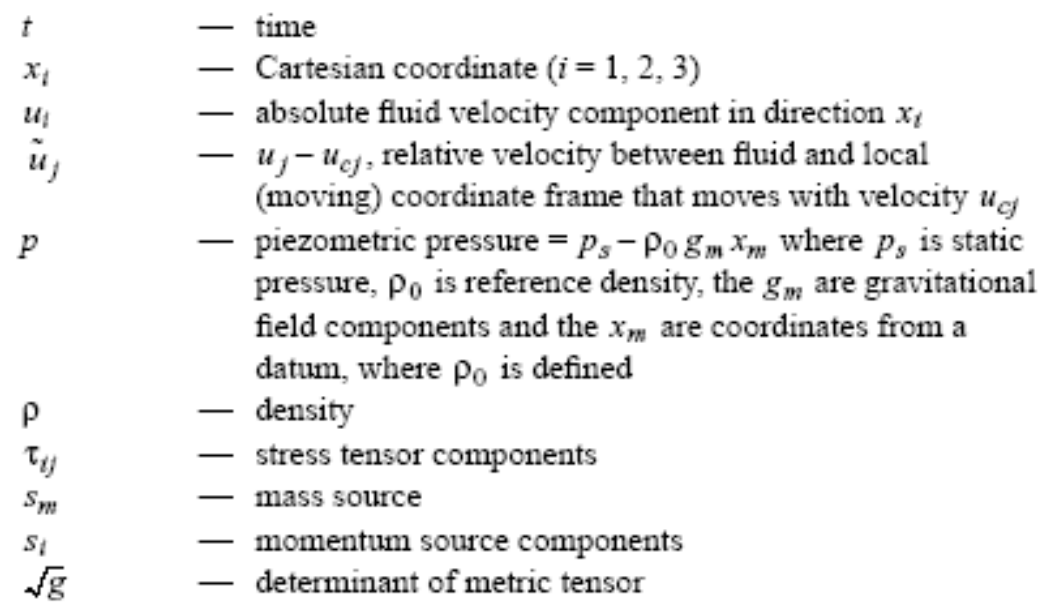

In the case of laminar flows, Star-CD caters for both Newtonian and nonNewtonian fluids that obey the following constitutive relation 


$$
\tau_{i j}=2 \mu s_{i j}-\frac{2}{3} \mu \frac{\partial u_{k}}{\partial x_{k}} \delta_{i j}
$$

where $\mu$ is the molecular dynamic fluid viscosity and, $\delta_{\mathrm{ij}}$, which is the Kronecker delta, is unity when $i=j$ and zero otherwise and finally, $s_{\mathrm{ij}}$, the rate of strain tensor, is given by

$$
s_{i j}=\frac{1}{2}\left(\frac{\partial u_{i}}{\partial x_{j}}+\frac{\partial u_{j}}{\partial x_{i}}\right)
$$

The governing equations are then discretize using the central-differencing scheme with a blending factor of 1 . Relaxation factors were set at 0.8 and 0.5 for the momentum in the vertical direction and pressure solver parameters respectively. The sparger model has 35420 fluid-cells with 320 partial boundaries.

\subsection{The CFD simulations}

The periodic cross-sectional contour-plots of the velocity-magnitude within the $2^{\text {nd }}$ L-bend of the sparger is shown in Fig. 2. It is observed at the middle-part of the pipe, the plots tend to exhibit horizontal-forms of contour-plots. This signifies the fluid-flow interaction around the boundary-regions of the nozzles.
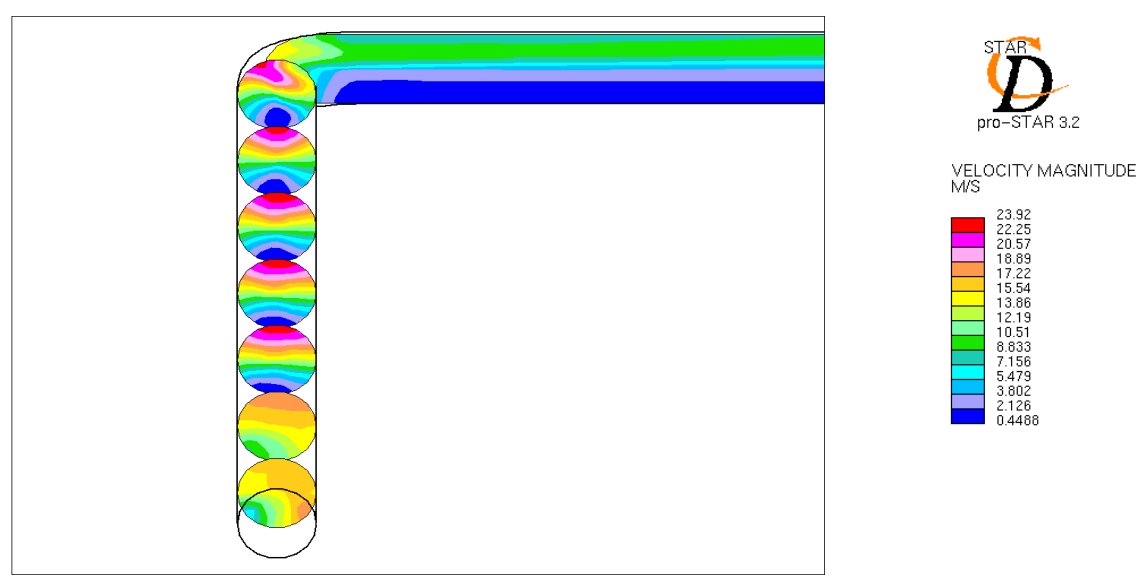

Figure 2: Periodic cross-sectional velocity-magnitude in contour-plot.

Figure 3 shows the particle-tracking plot with ribbon-widths of 0.1 model units and a twist-magnification of 1 . The ribbons are also plotted with meshplotting. This plot allows a much closer observation on the fluid-flow within the sparger at the $2^{\text {nd }}$ L-Bend. It is observed that the fluid flow is experiencing a vortex flow. 


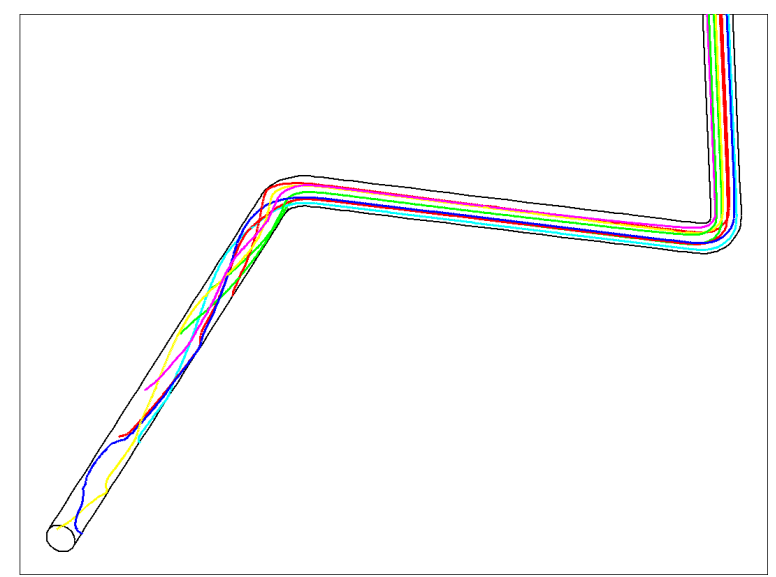

Figure 3: Particle-tracking in ribbon-form at $2^{\text {nd }}$ L-Bend of sparger in isometric view.

Figures 4 and 5 depicted the velocity and total pressure magnitude respectively for the whole pipe configuration. The fluid flow at the $1^{\text {st }}$ L-bend showing adverse pressure gradient indicating the occurrence of wake. However, downstream of the pipe before the $2^{\text {nd }}$ L-bend, the flow settles and the distribution shows that the flow tends to be uniform. On the other hand, this flow is not observed after the $2^{\text {nd }} \mathrm{L}$-bend.
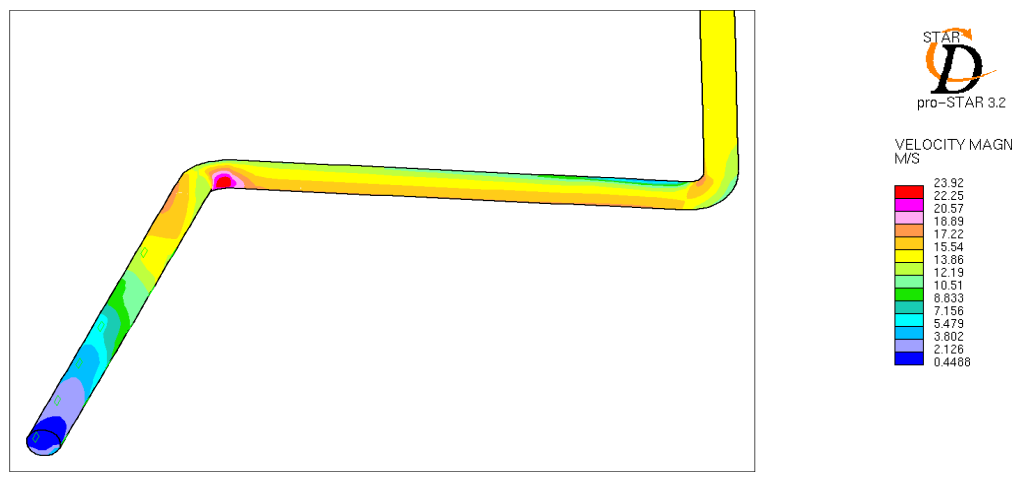

VELOCITY MAGNITUDE

Figure 4: The velocity distribution in the pipe.

This is clearly shown in Figure 6, where 20 contour-plots of the velocity magnitude at this section of the pipe are presented, that the flow is circulating.

\section{Discussion}

The results from the CFD simulations show that there exists a secondary flow in both bends, which is due to the dynamic pressure differences created on each 
bend. This phenomena is described in detail in many standard fluid mechanics textbooks, such as Munson et al. [4] and Çengel and Cimbala [5]. To reduce swirls and disturbances as observed in such flows, Munson et al. [4] suggest to use carefully designed guide vanes that help to direct the flow so that it stays uniform.
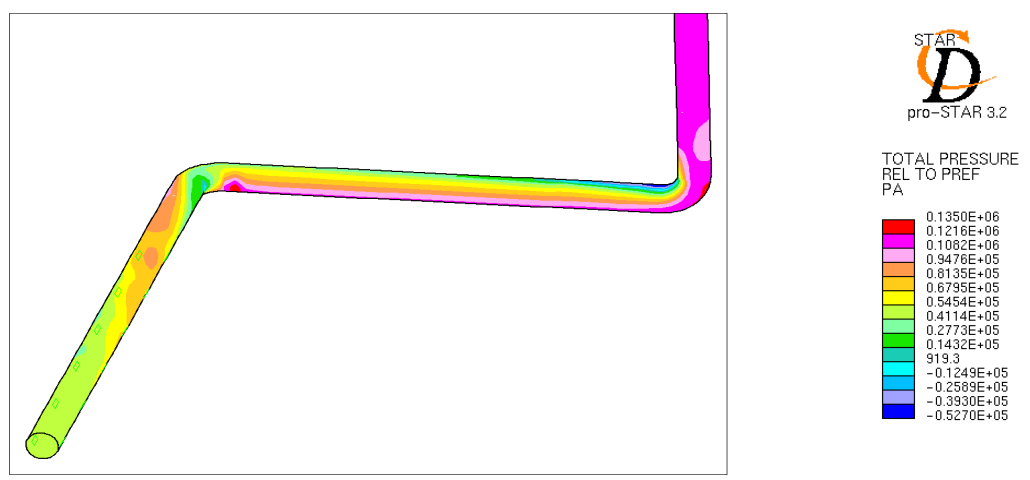

Figure 5: The total pressure distribution for the pipe.

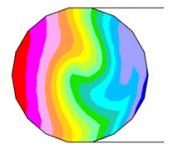

$1 \mathrm{~d}$

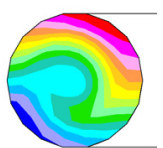

$6 \mathrm{~d}$

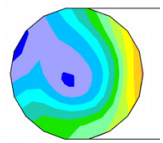

$11 \mathrm{~d}$

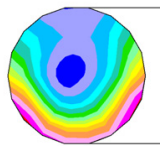

$16 \mathrm{~d}$

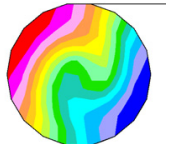

$2 \mathrm{~d}$

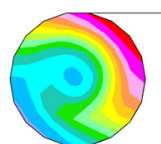

$7 \mathrm{~d}$

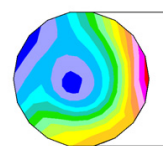

$12 \mathrm{~d}$

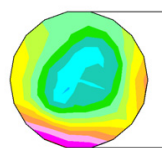

$17 \mathrm{~d}$

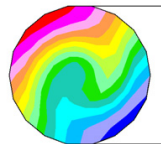

$3 \mathrm{~d}$

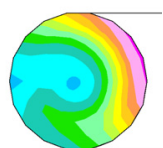

$8 \mathrm{~d}$

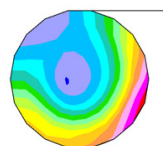

$13 \mathrm{~d}$

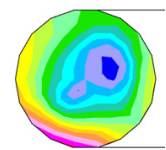

$18 \mathrm{~d}$

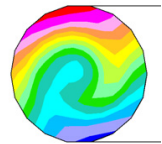

$4 d$

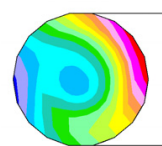

$9 \mathrm{~d}$

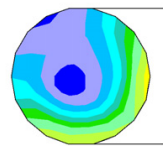

$14 \mathrm{~d}$

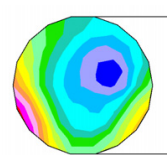

$19 d$

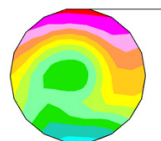

$5 \mathrm{~d}$

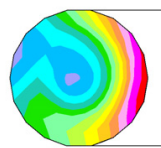

$10 \mathrm{~d}$

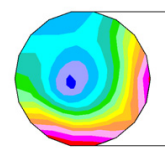

$15 d$

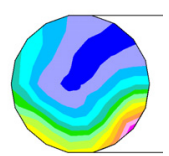

$20 d$

Figure 6: 20 contour-plots of the velocity magnitude at cross-sectional view.

Nevertheless, further upstream of the pipe bend without any guiding vanes, the velocity profiles should settle into a more uniform distribution (see Figure 4 and 5). The length of the pipe plays significant role in ensuring this statement as what has been concluded in Aroussi et al. [2] that the secondary flow patterns 
depends on the pipe geometry. However, this fully developed uniform flow pattern is not observed when the series of nozzles are included on the outlet arm. The flow becomes erratic whereby the streamlines follows a wave-like pattern (see Figure 3). This suggests that the fluid injected through the nozzles will not be of the same volume. In order to address this non-uniformity of fluid discharge from the nozzles, the location of the nozzle along the pipe and the size of the nozzle diameter could be varied as a function of the pipe length.

\section{Conclusions}

In order to obtain a uniform fluid discharge from the nozzles, the fluid flow past the second bend must be controlled so that the secondary flows will not occur. This suggests that the pipe configuration and design needs to be improved, perhaps by including guided vanes on the L-bends and adjusting the position of the nozzles and their diameter along the pipe.

\section{Acknowledgements}

Our gratitude to University Malaysia Sarawak for financial support (Fundamental research grant Code 02(55)/465/2004(202)), the Malaysian Ministry of Science, Technology and Innovation for student sponsorship (Almon Chai), and Komag USA (Malaysia) Ltd. for research collaboration.

\section{References}

[1] Morrison, G.L., Flow field development downstream of two in plane elbows. Proc. of the ASME Fluids Engineering Division Summer Meeting, Vancouver, British Columbia, Canada, Paper No: FEDSM97-3021, 1997.

[2] Aroussi, A., Roberts, J., and Rogers, P., Investigation of secondary flows in double pipe elbows: CFD study. Proc. of the $11^{\text {th }}$ Int. Symp. on Flow Visualizations, Notre Dame, Indiana, USA, Paper No: 39, 2004.

[3] Aroussi, A., Roberts, J., Rogers, P., and Box, G., Investigation of secondary flows in double pipe elbows: an experimental study. Proc. of the $11^{\text {th }}$ Int. Symp. on Flow Visualizations, Notre Dame, Indiana, USA, Paper No: 40, 2004.

[4] Munson, B. R., Young, D. F. and Okiishi, T. H., Fundamentals of Fluid Mechanics (4 ${ }^{\text {th }}$ Ed.), John Wiley \& Sons: USA, pp. 486, 2002.

[5] Çengel, Y. A. and Cimbala, J. M., Flow in pipes (Chapter 8). Topics in Fluid Mechanics: Fundamentals and Applications, McGraw-Hill: New York, pp. 335-354, 2005.

[6] Mendonça, F., Allen, R., Charentenay, J.D., and Lewis, M., Towards understanding LES and DES for Industrial Aeroacoustic Predictions. Proc. Int. Workshop on 'LES for Acoustics', Göttingen, Germany, 2002.

[7] Star-CD Methodology, Star-CD Ver. 3.15A, Computational Dynamics Ltd., UK, 2002. 